\title{
1,3,4-Oxadiazole N-Mannich Bases: Synthesis, Antimicrobial, and Anti-Proliferative Activities
}

\author{
Lamya H. Al-Wahaibi ${ }^{1}$, Ahmed A. B. Mohamed ${ }^{2}$, Samar S. Tawfik ${ }^{3}$, Hanan M. Hassan ${ }^{4}$ and Ali A. El-Emam ${ }^{2, *}$ \\ 1 Department of Chemistry, College of Sciences, Princess Nourah Bint Abdulrahman University, \\ Riyadh 11671, Saudi Arabia; lhalwahaibi@pnu.edu.sa \\ 2 Department of Medicinal Chemistry, Faculty of Pharmacy, Mansoura University, Mansoura 35516, Egypt; \\ ahmed_smt@yahoo.com \\ 3 Department of Pharmaceutical Organic Chemistry, Faculty of Pharmacy, Mansoura University, \\ Mansoura 35516, Egypt; samarsamir1984@gmail.com \\ 4 Department of Pharmacology and Biochemistry, Faculty of Pharmacy, Delta University for Science and \\ Technology, International Costal Road, Gamasa City, Mansoura 11152, Egypt; hananhafila@hotmail.com \\ * Correspondence: elemam@mans.edu.eg; Tel.: +20-50-2258087
}

Citation: Al-Wahaibi, L.H.; Mohamed, A.A.B.; Tawfik, S.S.; Hassan, H.M.; El-Emam, A.A. 1,3,4-Oxadiazole N-Mannich Bases: Synthesis, Antimicrobial, and Anti-Proliferative Activities. Molecules 2021, 26, 2110. https:// doi.org/10.3390/molecules26082110

Academic Editor: Athina Geronikaki

Received: 5 March 2021

Accepted: 31 March 2021

Published: 7 April 2021

Publisher's Note: MDPI stays neutral with regard to jurisdictional claims in published maps and institutional affiliations.

Copyright: (C) 2021 by the authors. Licensee MDPI, Basel, Switzerland. This article is an open access article distributed under the terms and conditions of the Creative Commons Attribution (CC BY) license (https:// creativecommons.org/licenses/by/ $4.0 /)$.

\begin{abstract}
The reaction of 5-(3,4-dimethoxyphenyl)-1,3,4-oxadiazole-2(3H)-thione 3 with formaldehyde solution and primary aromatic amines or 1-substituted piperazines, in ethanol at room temperature yielded the corresponding N-Mannich bases 3-arylaminomethyl-5-(3,4-dimethoxyphenyl)-1,3,4oxadiazole-2(3H)-thiones 4 a-1 or 3-[(4-substituted piperazin-1-yl)methyl]-5-(3,4-dimethoxyphenyl)1,3,4-oxadiazole-2(3H)-thiones 5a-d, respectively. The in vitro inhibitory activity of compounds 4a-1 and 5a-d was assessed against pathogenic Gram-positive, Gram-negative bacteria, and the yeast-like pathogenic fungus Candida albicans. The piperazinomethyl derivatives $\mathbf{5} \mathbf{c}$ and $\mathbf{5} \mathbf{d}$ displayed broad-spectrum antibacterial activities the minimal inhibitory concentration (MIC) $0.5-8 \mu \mathrm{g} / \mathrm{mL}$ ) and compounds $4 \mathbf{j}, \mathbf{4 1}, \mathbf{5 a}$, and $\mathbf{5 b}$ showed potent activity against the tested Gram-positive bacteria. In addition, the anti-proliferative activity of the compounds was evaluated against prostate cancer (PC3), human colorectal cancer (HCT-116), human hepatocellular carcinoma (HePG-2), human epithelioid carcinoma (HeLa), and human breast cancer (MCF7) cell lines. The optimum anti-proliferative activity was attained by compounds $41,5 a, 5 c$, and $5 \mathrm{~d}$.
\end{abstract}

Keywords: 1,3,4-oxadiazoles; N-Mannich bases; antimicrobial activity; anti-proliferative activity

\section{Introduction}

The 1,3,4-Oxadiazoles are an important class of heterocyclic compounds with diverse pharmacological properties [1-3]. The 1,3,4-Oxadiazole nucleus represents an essential building unit in several drugs including the broad spectrum antibacterial drug furamizole [4], the antiretroviral drug raltegravir [5], the anticancer agent zibotentan [6], the antiobesity/antidiabetic agent AZD 3988 [7], and the antihypertensive drugs tiodazosin [8], and nesadipil [9].

In addition, there is a growing interest in the chemotherapeutic activities of 2,5disubstituted-1,3,4-oxadiazoles as antibacterial [10-12], antifungal [13-15], antitubercular [16,17], and antiviral [18-21] agents. Moreover, 1,3,4-Oxadiazole-2(3H)-thiones, their thioether derivatives and 3-aminomethyl analogues ( $N$-Mannich bases) are the most interesting for their anticancer activities [21,22]. The 1,3,4-Oxadiazole derivatives exert their anticancer activities via different mechanisms, such as targeting epidermal growth factor receptors (EGFR) [23], vascular endothelial growth factor receptors (VEGF) [24], focal-adhesion kinase (FAK) [25,26], histone deacetylases (HDAC) [27,28], methionine aminopeptidase (MetAP) [29], NF- $\mathrm{B}$ (nuclear factor $\kappa \mathrm{B}$ ) [30], poly(ADP-ribose) polymerase (PARP-1) [31], thymidine phosphorylase (TP) [32], telomerase [33], thymidylate synthase (TS) [34], zinc-finger protein 143 (ZNF143) [35], and tubulin polymerase [36]. 
Furthermore, 1,3,4-oxadiazoles were proved to exhibit potent anti-inflammatory [37-39], antioxidant [40], antidiabetic [41,42], and monoamine oxidase (MAO) inhibitory activities [43,44]. Besides, 1,3,4-oxadiazole derivatives are highly attractive compounds in the development of organic light-emitting diodes (OLEDs) [45,46].

Furthermore, 3,4-dimethoxyphenyl moiety represents an essential motif in various chemotherapeutic agents with anticancer [47-52], antibacterial [53,54], and antiviral [55] activities.

Motivated by the above-mentioned findings and following an ongoing interest in the pharmacological [56] and structural properties [57-59] of 1,3,4-oxadiazole-2(3H)-thione $\mathrm{N}$-Mannich bases, we report herein the synthesis, characterization, antibacterial, antifungal, and anti-proliferative activities of related series of 5-(3,4-dimethoxyphenyl)-1,3,4oxadiazole-2(3H)-thione $\mathrm{N}$-Mannich bases.

\section{Results and Discussion}

\subsection{Chemical Synthesis}

The 3,4-Dimethoxybenzohydrazide 2 was prepared from the commercially-available methyl 3,4-dimethoxybenzoate 1 via treatment with hydrazine in ethanol [60]. 1,3,4Oxadiazole-2(3H)-thione 3 was obtained via reaction of the carbohydrazide 2 with carbon disulfide in ethanolic potassium hydroxide as previously described [61]. Furthermore, 1,3,4Oxadiazole-2(3H)-thiones were reported to undergo aminomethylation through reaction with primary aromatic amines and formaldehyde to yield the corresponding N-Mannich bases [62]. Consequently, treatment of $\mathbf{3}$ with formaldehyde solution and various primary aromatic amines or 1-substituted piperazines, in ethanol at room temperature yielded their corresponding 3-arylaminomethyl $\mathbf{4 a - 1}$ or 3-piperazinomethyl $\mathbf{5 a}-\mathbf{d} \mathrm{N}$-Mannich bases, respectively, in good yields (Scheme 1, Table 1).

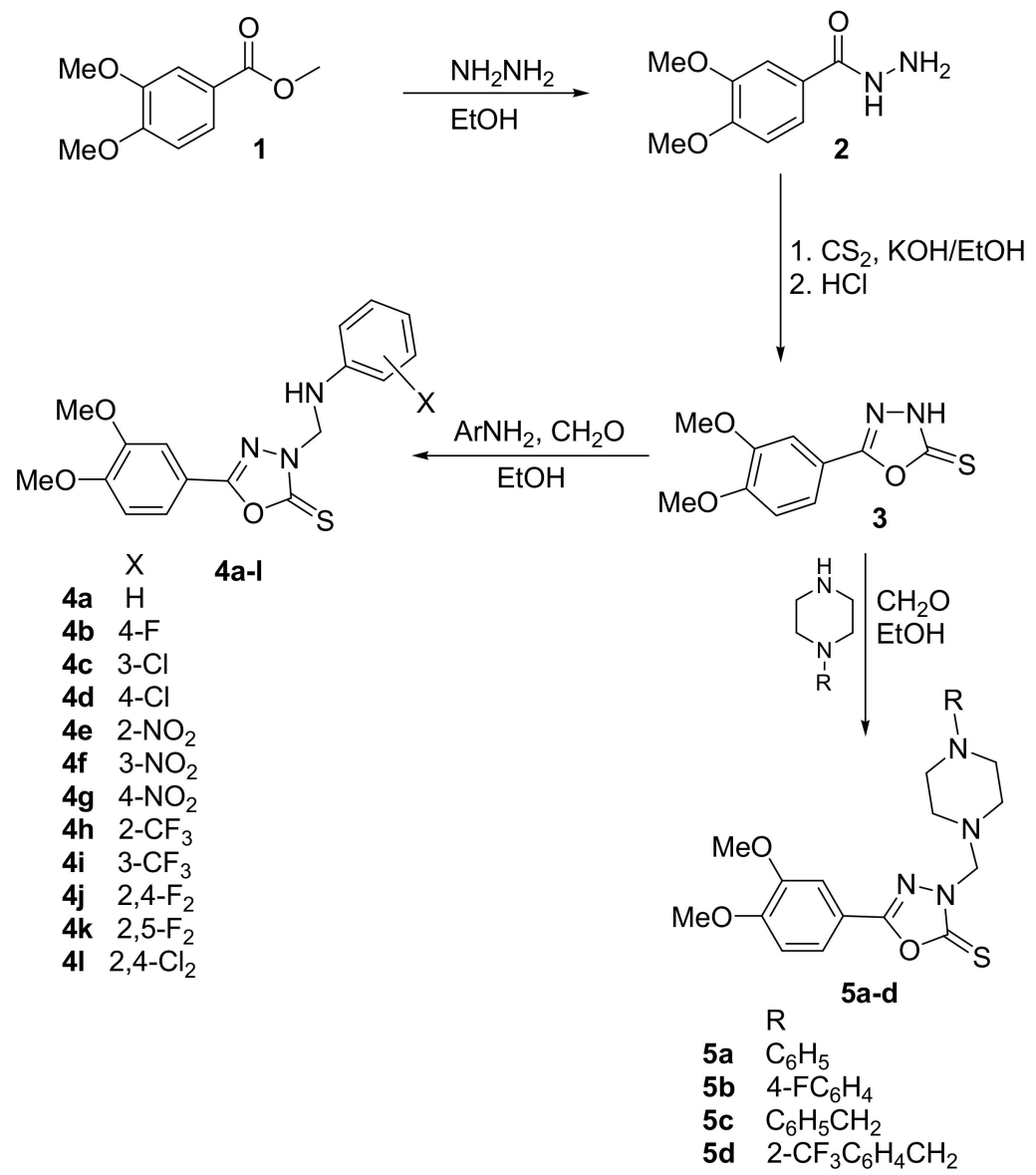

Scheme 1. Synthesis of compounds $4 \mathbf{a}-\mathbf{1}$ and $5 \mathbf{a}-\mathbf{d}$. 
Table 1. Crystallization solvents, melting points (M.P.), yield percentages, molecular (Mol.) formulae, and molecular weights (Wt.) of compounds $4 \mathbf{a}-\mathbf{1}$ and $\mathbf{5 a}-\mathbf{d}$.

\begin{tabular}{cccccc}
\hline Compound No. & $\mathbf{X} / \mathbf{R}$ & Crystallization Solvents & M.P. $\left({ }^{\circ} \mathbf{C}\right)$ & Yield (\%) & Mol. Formula (Mol. Wt.) \\
\hline $\mathbf{4 a}$ & $\mathrm{H}$ & $\mathrm{EtOH} / \mathrm{H}_{2} \mathrm{O}$ & $147-149$ & 82 & $\mathrm{C}_{17} \mathrm{H}_{17} \mathrm{~N}_{3} \mathrm{O}_{3} \mathrm{~S}(343.40)$ \\
$\mathbf{4} \mathbf{b}$ & $4-\mathrm{F}$ & $\mathrm{EtOH} / \mathrm{H}_{2} \mathrm{O}$ & $135-137$ & 84 & $\mathrm{C}_{17} \mathrm{H}_{16} \mathrm{FN}_{3} \mathrm{O}_{3} \mathrm{~S}(361.39)$ \\
$\mathbf{4} \mathbf{c}$ & $3-\mathrm{Cl}$ & $\mathrm{EtOH}$ & $143-145$ & 77 & $\mathrm{C}_{17} \mathrm{H}_{16} \mathrm{ClN}_{3} \mathrm{O}_{3} \mathrm{~S}(377.85)$ \\
$\mathbf{4}$ & $4-\mathrm{Cl}$ & $\mathrm{EtOH}$ & $166-168$ & 79 & $\mathrm{C}_{17} \mathrm{H}_{16} \mathrm{ClN}_{3} \mathrm{O}_{3} \mathrm{~S}(377.85)$ \\
$\mathbf{4} \mathbf{e}$ & $2-\mathrm{NO}_{2}$ & $\mathrm{EtOH} / \mathrm{CHCl}$ & $211-213$ & 88 & $\mathrm{C}_{17} \mathrm{H}_{16} \mathrm{~N}_{4} \mathrm{O}_{5} \mathrm{~S}(388.40)$ \\
$\mathbf{4} \mathbf{f}$ & $3-\mathrm{NO}_{2}$ & $\mathrm{EtOH} / \mathrm{CHCl}_{3}$ & $181-183$ & 85 & $\mathrm{C}_{17} \mathrm{H}_{16} \mathrm{~N}_{4} \mathrm{O}_{5} \mathrm{~S}(388.40)$ \\
$\mathbf{4} \mathbf{g}$ & $4-\mathrm{NO}_{2}$ & $\mathrm{EtOH} / \mathrm{CHCl}_{3}$ & $222-224$ & 92 & $\mathrm{C}_{17} \mathrm{H}_{16} \mathrm{~N}_{4} \mathrm{O}_{5} \mathrm{~S}(388.40)$ \\
$\mathbf{4}$ & $2-\mathrm{CF}_{3}$ & $\mathrm{EtOH} / \mathrm{H}_{2} \mathrm{O}$ & $220-222$ & 90 & $\mathrm{C}_{18} \mathrm{H}_{16} \mathrm{~F}_{3} \mathrm{~N}_{3} \mathrm{O}_{3} \mathrm{~S}(411.40)$ \\
$\mathbf{4} \mathbf{i}$ & $3-\mathrm{CF}_{3}$ & $\mathrm{EtOH} / \mathrm{H}_{2} \mathrm{O}$ & $206-208$ & 86 & $\mathrm{C}_{18} \mathrm{H}_{16} \mathrm{~F}_{3} \mathrm{~N}_{3} \mathrm{O}_{3} \mathrm{~S}(411.40)$ \\
$\mathbf{4} \mathbf{j}$ & $2,4-\mathrm{F}_{2}$ & $\mathrm{EtOH}$ & $169-171$ & 92 & $\mathrm{C}_{17} \mathrm{H}_{15} \mathrm{~F}_{2} \mathrm{~N}_{3} \mathrm{O}_{3} \mathrm{~S}(379.38)$ \\
$\mathbf{4}$ & $2,5-\mathrm{F}_{2}$ & $\mathrm{EtOH}$ & $212-214$ & 90 & $\mathrm{C}_{17} \mathrm{H}_{15} \mathrm{~F}_{2} \mathrm{~N}_{3} \mathrm{O}_{3} \mathrm{~S}(379.38)$ \\
$\mathbf{4}$ & $2,4-\mathrm{Cl}_{2}$ & $\mathrm{EtOH}$ & $227-229$ & 94 & $\mathrm{C}_{17} \mathrm{H}_{15} \mathrm{Cl}_{2} \mathrm{~N}_{3} \mathrm{O}_{3} \mathrm{~S}(412.29)$ \\
$\mathbf{5}$ & $\mathrm{C}_{6} \mathrm{H}_{5}$ & $\mathrm{EtOH}$ & $151-153$ & 85 & $\mathrm{C}_{21} \mathrm{H}_{24} \mathrm{~N}_{4} \mathrm{O}_{3} \mathrm{~S}(412.51)$ \\
$\mathbf{5 b}$ & $4-\mathrm{FC}_{6} \mathrm{H}_{4}$ & $\mathrm{EtOH}$ & $118-120$ & 78 & $\mathrm{C}_{21} \mathrm{H}_{23} \mathrm{FN}_{4} \mathrm{O}_{3} \mathrm{~S}(430.50)$ \\
$\mathbf{5}$ & $\mathrm{C}_{6} \mathrm{H}_{5} \mathrm{CH}_{2}$ & $\mathrm{EtOH} / \mathrm{H}_{2} \mathrm{O}$ & $121-123$ & 75 & $\mathrm{C}_{22} \mathrm{H}_{26} \mathrm{~N}_{4} \mathrm{O}_{3} \mathrm{~S}(426.53)$ \\
$\mathbf{5 d}$ & $2-\mathrm{CF}_{3} \mathrm{C}_{6} \mathrm{H}_{4} \mathrm{CH}_{2}$ & $\mathrm{EtOH}$ & $141-143$ & 89 & $\mathrm{C}_{23} \mathrm{H}_{25} \mathrm{~F}_{3} \mathrm{~N}_{4} \mathrm{O}_{3} \mathrm{~S}(494.53)$ \\
\hline
\end{tabular}

The structures of compounds $\mathbf{4 a}-\mathbf{1}$ and $\mathbf{5 a}-\mathbf{d}$ were confirmed by elemental analyses, ${ }^{1} \mathrm{H}$ NMR and ${ }^{13} \mathrm{C}$ NMR spectral data.

\subsection{In Vitro Antibacterial and Antifungal Activities}

The in vitro growth inhibitory activity of compounds $\mathbf{4 a - 1}$ and $\mathbf{5 a - d}$ was evaluated towards the standard Gram-positive bacterial strains Staphylococcus aureus American type culture collection (ATCC) 6571, Bacillus subtilis ATCC 5256 and Micrococcus luteus ATCC 27141, Gram-negative bacterial strains Escherichia coli ATCC 8726, and Pseudomonas aeruginosa ATCC 27853, and the yeast-like pathogenic fungus Candida albicans MTCC 227. The initial screening was performed by the semi-quantitative agar-disc diffusion method using Müller-Hinton agar medium [63]. The results of the preliminary screening of compounds 4a- -1 and $5 \mathbf{a}-\mathbf{d}(200 \mu \mathrm{g} /$ disc); the antibacterial antibiotics Gentamicin sulfate, Ampicillin trihydrate, and the antifungal drug Clotrimazole $(100 \mu \mathrm{g} /$ disc $)$; and the calculated $\log P$ values $(C \log P)$ are depicted in Table 2.

The results revealed that potent antibacterial activity was displayed by the compounds $4 \mathbf{j}, \mathbf{4} \mathbf{1}, \mathbf{5 a}, \mathbf{5 b}, \mathbf{5 c}$, and $\mathbf{5 d}$, which displayed growth inhibition zones $\geq 18 \mathrm{~mm}$ against one or more of the tested microorganisms. In addition, compounds $4 a, 4 b, 4 c, 4 d, 4 h, 4 i$, and 4k showed medium activity (growth inhibition zones $14-17 \mathrm{~mm}$ ) and compounds $4 \mathbf{e}, 4 \mathrm{f}$, and $4 \mathrm{~g}$ showed poor antibacterial activity (growth inhibition zones $10-13 \mathrm{~mm}$ ) against the tested microorganisms. In general, the activity against the tested Gram-positive bacteria is higher than the activity against the tested Gram-negative bacteria. The optimal antibacterial activity was attained by compounds $5 \mathbf{c}$ and $\mathbf{5 d}$, which showed potent and broad-spectrum antibacterial activity against all the tested bacterial strains. The inhibitory activity of the compounds against Candida albicans was generally lower than their antibacterial activity, compounds $\mathbf{4 g}$ displayed medium activity, and all other compounds were either poorly active or inactive compared with Clotrimazole.

The minimal inhibitory concentrations (MICs) of the most active compounds $\mathbf{4 j}, \mathbf{4 1}, \mathbf{5 a}$, $\mathbf{5 b}, \mathbf{5 c}$, and $\mathbf{5 d}$, and the antibacterial antibiotics Gentamicin sulfate, Ampicillin trihydrate, and the antifungal drug Clotrimazole were determined by the microdilution susceptibility method in Müller-Hinton broth and Sabouraud liquid medium [64]. The MIC values were highly consistent with their growth inhibition zones. 
Table 2. In vitro activity of compounds $4 \mathbf{a}-\mathbf{1}$ and $\mathbf{5 a}-\mathbf{d}(200 \mu \mathrm{g} / 8 \mathrm{~mm}$ disc); the broad-spectrum antibacterial drugs Gentamicin sulfate, Ampicillin trihydrate, and the antifungal drug Clotrimazole (100 $\mu \mathrm{g} / 8 \mathrm{~mm}$ disc) against Staphylococcus aureus American type culture collection (ATCC) 6571 (SA), Bacillus subtilis ATCC 5256 (BS), Micrococcus luteus ATCC 27,141 (ML), Escherichia coli ATCC 8726 (EC), Pseudomonas aeruginosa ATCC 27,853 (PA), and the yeast-like pathogenic fungus Candida albicans MTCC 227 (CA).

\begin{tabular}{|c|c|c|c|c|c|c|c|}
\hline \multirow{2}{*}{$\begin{array}{c}\text { Comp. } \\
\text { No. }\end{array}$} & \multirow{2}{*}{$C \log P^{c}$} & \multicolumn{6}{|c|}{ Diameter of Growth Inhibition Zone $(\mathrm{mm})^{a}$} \\
\hline & & SA & BS & ML & EC & PA & CA \\
\hline $4 a$ & 3.701 & 14 & 13 & 15 & - & - & 10 \\
\hline $4 b$ & 4.146 & 15 & 16 & 17 & - & - & 12 \\
\hline $4 c$ & 4.716 & 14 & 17 & 13 & - & - & - \\
\hline $4 d$ & 4.716 & 15 & 13 & 14 & - & - & - \\
\hline $4 e$ & 4.242 & 12 & 11 & 10 & - & - & 13 \\
\hline $4 f$ & 4.092 & 11 & 12 & - & - & - & 12 \\
\hline $4 g$ & 4.092 & 13 & 12 & 11 & - & - & 16 \\
\hline $4 h$ & 5.113 & 15 & 12 & - & - & - & - \\
\hline $4 i$ & 5.113 & 16 & 12 & - & - & - & - \\
\hline $4 j$ & 4.395 & $19(4)^{b}$ & 12 & 16 & 12 & 13 & 11 \\
\hline $4 k$ & 4.395 & 17 & 14 & 15 & 14 & - & 12 \\
\hline 41 & 5.535 & $19(4)^{b}$ & $21(2)^{b}$ & $18(16)^{b}$ & 15 & 12 & 11 \\
\hline $5 a$ & 3.789 & $23(1)^{b}$ & $26(1)^{b}$ & $21(1)^{b}$ & 14 & 13 & - \\
\hline $5 b$ & 4.103 & $20(8)^{b}$ & $23(1)^{b}$ & $20(1)^{b}$ & 16 & 17 & - \\
\hline $5 c$ & 4.712 & $26(1)^{b}$ & $30(0.5)^{b}$ & $22(1)^{b}$ & $19(4)^{b}$ & $18(4)^{b}$ & - \\
\hline $5 d$ & 5.595 & $28(1)^{b}$ & $29(1)^{b}$ & $26(1)^{b}$ & $22(1)^{b}$ & $20(2)^{b}$ & - \\
\hline \multicolumn{2}{|c|}{ Gentamicin sulfate } & $27(1)^{b}$ & $26(2)^{b}$ & $20(2)^{b}$ & $22(0.5)^{b}$ & $21(0.5)^{b}$ & NT \\
\hline \multicolumn{2}{|c|}{ Ampicillin trihydrate } & $22(2)^{b}$ & $23(1)^{b}$ & $20(2)^{b}$ & $16(8)^{b}$ & $16(8)^{b}$ & NT \\
\hline \multicolumn{2}{|c|}{ Clotrimazole } & NT & NT & NT & NT & NT & $21(4)^{b}$ \\
\hline
\end{tabular}

a $(-)$ : inactive (inhibition zone $<10 \mathrm{~mm}$ ), ${ }^{\mathrm{b}}$ Figures shown in parentheses represent the minimal inhibitory concentration (MIC) values $(\mu \mathrm{g} / \mathrm{mL}),{ }^{c}$ Calculated using the CS ChemOffice Ultra version 8.0, CambridgeSoft, Cambridge, MA, USA), NT: not tested. SA, Staphylococcus aureus; BS, Bacillus subtilis; ML, Micrococcus luteus; EC, Escherichia coli; PA, Pseudomonas aeruginosa; CA, Candida albicans. High activity ( $>18 \mathrm{~mm})$ values have been bolded for emphasis.

Based on the results of the antibacterial activity, it could be concluded that the activity of the piperazinomethyl $\mathrm{N}$-Mannich bases $\mathbf{5 a - d}$ is superior to their arylaminomethyl analogues $4 a-1$. Considering the anilinomethyl analogue $4 \mathbf{a}$ as the basic structure of the arylaminomethyl analogues $\mathbf{4 a}-\mathbf{1}$, the antibacterial activity of the monohalo derivatives $\mathbf{4 b - d}$ was slightly improved against the tested Gram-positive bacteria. Meanwhile, the antibacterial activity of the nitro derivatives $4 \mathbf{e}-\mathbf{g}$ was greatly declined with general improvement of the antifungal activity. Despite the high lipophilicity of the trifluoromethyl derivatives $4 \mathrm{~h}$ and $4 \mathbf{i}$, the compounds only retained moderate activity against Staphylococcus aureus and lacked activity against the tested Gram-positive bacteria and Candida albicans. Introduction a difluoro- or dichlorophenyl moieties (compounds $4 \mathbf{j}-\mathbf{1}$ ) greatly enhanced the Gram-positive antibacterial activity and these derivatives endowed moderate or marginal activity against the tested Gram-negative bacteria and Candida albicans.

The replacement of the arylaminomethyl moiety with a piperazinomethyl moiety greatly enhanced the antibacterial activity and the piperazinomethyl derivatives $\mathbf{5 a}-\mathbf{d}$ exhibited higher potency and broader antibacterial spectrum compared to their arylaminomethyl analogues $4 \mathbf{a}-\mathbf{l}$, with no antifungal activity. In addition, the antibacterial activity of the piperazinomethyl derivatives seems correlated to their lipophilicity as the optimum antibacterial activity against the tested Gram-negative bacteria was displayed by compounds $5 \mathrm{c}$ and $5 \mathrm{~d}$.

\subsection{In Vitro Anti-proliferative Activity}

The 3-(4,5-dimethylthiazol-2-yl)-2,5-diphenyltetrazolium bromide (MTT) colorimetric assay $[65,66]$ was adopted to evaluate the in vitro anti-proliferative activity of compounds 
4a-1 and 5a-d against five human cancer cell lines namely; prostate cancer (PC3), human colorectal cancer (HCT-116), human hepatocellular carcinoma (HePG-2), human epithelioid carcinoma (HeLa) and human breast cancer (MCF7) cell lines. The results of the antiproliferative activity of compounds $4 \mathbf{a}-\mathbf{1}, \mathbf{5 a}-\mathbf{d}$, and the anticancer drug Doxorubicin [67] are displayed in Table 3.

Table 3. In vitro anti-proliferative activity of the tested compounds $4 \mathbf{a}-\mathbf{1}, \mathbf{5 a}-\mathbf{d}$, and Doxorubicin expressed as $\mathrm{IC}_{50}$ values against prostate cancer (PC3), human colorectal cancer (HCT-116), human hepatocellular carcinoma (HePG-2), human epithelioid carcinoma (HeLa), and MCF7 human cancer cell line.

\begin{tabular}{|c|c|c|c|c|c|}
\hline \multirow{2}{*}{$\begin{array}{c}\text { Comp. } \\
\text { No. }\end{array}$} & \multicolumn{5}{|c|}{$\mathrm{IC}_{50}(\mu \mathrm{M})^{\mathrm{a}}$} \\
\hline & PC3 & HCT-116 & HePG-2 & HeLa & MCF7 \\
\hline $4 a$ & $63.94 \pm 3.8$ & $53.17 \pm 3.3$ & $47.23 \pm 3.1$ & $28.31 \pm 2.0$ & $55.34 \pm 2.8$ \\
\hline $4 b$ & $71.80 \pm 4.0$ & $77.52 \pm 4.1$ & $56.34 \pm 3.3$ & $49.47 \pm 3.0$ & $68.26 \pm 3.4$ \\
\hline $4 c$ & $84.52 \pm 4.8$ & $39.44 \pm 2.6$ & $78.86 \pm 4.1$ & $44.69 \pm 2.8$ & $33.86 \pm 2.3$ \\
\hline $4 d$ & $78.35 \pm 4.5$ & $48.30 \pm 3.0$ & $73.80 \pm 3.9$ & $54.02 \pm 3.2$ & $40.52 \pm 2.5$ \\
\hline $4 e$ & $>100$ & $92.11 \pm 4.9$ & $>100$ & $75.61 \pm 3.9$ & $88.33 \pm 3.9$ \\
\hline $4 f$ & $74.67 \pm 4.4$ & $69.38 \pm 3.8$ & $58.41 \pm 3.5$ & $57.26 \pm 2.5$ & $65.35 \pm 3.2$ \\
\hline $4 g$ & $>100$ & $92.11 \pm 4.9$ & $>100$ & $75.61 \pm 3.9$ & $88.33 \pm 3.9$ \\
\hline $4 \mathrm{~h}$ & $59.48 \pm 3.5$ & $35.01 \pm 2.7$ & $42.74 \pm 2.9$ & $31.72 \pm 2.2$ & $29.10 \pm 2.1$ \\
\hline $4 i$ & $59.48 \pm 3.5$ & $35.01 \pm 2.7$ & $42.74 \pm 2.9$ & $31.72 \pm 2.2$ & $29.10 \pm 2.1$ \\
\hline $4 j$ & $95.61 \pm 5.1$ & $64.07 \pm 3.5$ & $86.45 \pm 4.5$ & $61.98 \pm 3.5$ & $59.87 \pm 2.7$ \\
\hline $4 \mathrm{k}$ & $75.22 \pm 4.1$ & $61.07 \pm 3.4$ & $77.40 \pm 3.6$ & $49.55 \pm 3.2$ & $29.56 \pm 3.6$ \\
\hline 41 & $34.60 \pm 2.3$ & $19.95 \pm 1.8$ & $17.42 \pm 1.4$ & $10.96 \pm 1.1$ & $12.97 \pm 1.0$ \\
\hline $5 a$ & $52.53 \pm 3.3$ & $27.49 \pm 2.3$ & $36.08 \pm 2.5$ & $24.09 \pm 1.8$ & $17.80 \pm 1.3$ \\
\hline $5 b$ & $>100$ & $89.26 \pm 4.6$ & $91.78 \pm 4.9$ & $67.53 \pm 3.7$ & $79.16 \pm 3.6$ \\
\hline $5 c$ & $23.92 \pm 1.9$ & $14.69 \pm 1.2$ & $11.93 \pm 1.0$ & $9.50 \pm 0.8$ & $6.49 \pm 0.4$ \\
\hline $5 d$ & $38.02 \pm 2.5$ & $32.81 \pm 2.6$ & $22.91 \pm 1.6$ & $18.37 \pm 1.4$ & $24.33 \pm 1.9$ \\
\hline Doxorubicin & $8.87 \pm 0.6$ & $5.23 \pm 0.3$ & $4.50 \pm 0.2$ & $5.57 \pm 0.4$ & $4.17 \pm 0.2$ \\
\hline
\end{tabular}

${ }^{\mathrm{a}} \mathrm{IC} 50$ values presented as the mean \pm SD of three separate determinations. Significant values $(<25 \mu \mathrm{m})$ have been bolded for emphasis.

According to the anti-proliferative activity results, the tested compounds exhibited variable degrees of activity against the tested cancer cell lines. In general, the activity against MCF-7 and HeLa seems higher than PC-3, HCT-116, and HePG-2. In addition, the activity of the piperazinomethyl analogues is higher than their arylaminomethyl analogues. The optimal activity was attained by compounds $41,5 a, 5 c$, and $5 \mathrm{~d}$ with $\mathrm{IC}_{50}<25 \mu \mathrm{M}$ against the tested cell lines.

Within the arylaminomethyl analogues $4 \mathbf{a}-1$, the compounds were inactive against PC3 cell lines $\left(\mathrm{IC}_{50}>100 \mu \mathrm{M}\right)$ or weakly active $\left(\mathrm{IC}_{50}=51-100 \mu \mathrm{M}\right)$ except compounds 41, which retained moderate activity $\left(\mathrm{IC}_{50} 34.60 \mu \mathrm{M}\right)$. The anti-proliferative activity of the arylaminomethyl analogues $4 \mathbf{a}-\mathbf{1}$ seems dependent on the aryl substituents, the 2,4dichlorophenyl is the optimal substituents while the nitrophenyl substitution at 2- or 4- position (compounds $4 \mathbf{e}, \mathbf{g}$ ) greatly deteriorated the anti-proliferative activity. The anilinomethyl analogue 4a, monohalophenylaminomethyl analogues $4 \mathbf{b}-\mathbf{d}$, the trifluoromethylphenyl

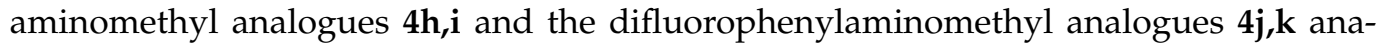
logues only retained moderate anti-proliferative activity $\left(\mathrm{IC}_{50}=26-50 \mu \mathrm{M}\right)$ or poor activity $\left(\mathrm{IC}_{50}=51-100 \mu \mathrm{M}\right)$.

Concerning the anti-proliferative activity of the piperazinomethyl derivatives $\mathbf{5 a - d}$, the 4-phenylpiperazinomethyl derivative 5a exhibited potent activity against HeLa and MFC7 cell lines, moderate activity against HCT-116 cell lines and weak activity against PC3 cell lines. Substitution of the phenyl group of compound 5a with 4-fluorophenyl group (compound 5b) dramatically deteriorated the anti-proliferative activity. The optimum antiproliferative activity was attained by the 4-benzylpiperazinomethyl derivative $5 c$, which showed potent activity against all the tested cell lines. Replacement of the benzyl 
group of compound $\mathbf{5 c}$ with 2-trifluoromethylbenzyl group (compound $\mathbf{5 d}$ ) reduced the anti-proliferative activity against the tested cancer cell lines.

\section{Materials and Methods}

\subsection{General Information}

Melting points $\left({ }^{\circ} \mathrm{C}\right.$, uncorrected) were determined in open glass capillaries using a Barnstead 9100 electro-thermal melting point apparatus. Nuclear magnetic resonance (NMR) spectra were determined in DMSO- $d_{6}$ on a JEOL ECA $500 \mathrm{III}$ at $500.16 \mathrm{MHz}$ for ${ }^{1} \mathrm{H}$ and $125.77 \mathrm{MHz}$ for ${ }^{13} \mathrm{C}$. Elemental analyses $(\mathrm{C}, \mathrm{H}, \mathrm{N}$, and $\mathrm{S})$ were in agreement with the proposed structures within $\pm 0.4 \%$ of the theoretical values (Table S1). Monitoring of the reactions and checking of the purity of the final products were carried out by thin layer chromatography (TLC) using silica gel pre-coated aluminum sheets $\left(60 \mathrm{~F}_{254}\right.$; Merck) and visualization with ultraviolet light (UV) at 365 and $254 \mathrm{~nm}$ and/or stained with an anisaldehyde solution and a phosphomolybdic acid solution. All chemicals and solvents were purchased from Alfa Aesar (Germany), and used without additional purification. The reference drugs Gentamicin sulfate (CAS 1405-41-0), Ampicillin trihydrate (CAS 7177-48-2), Clotrimazole (CAS 23593-75-1) and Doxorubicin (CAS 23214-92-8) were purchased from Sigma-Aldrich Chemie GmbH (Germany). Compound 3 was prepared according to the previously reported procedure [61].

3.2. Synthesis of 3-(Arylaminomethyl)-5-(3,4-Dimethoxyphenyl)-1,3,4-Oxadiazole-2(3H)-Thiones 4a-l and 3-[(4-Substituted Piperazin-1-yl)Methyl]-5-(3,4-Dimethoxyphenyl\}-1,3,4-Oxadiazole2(3H)-Thiones $5 \boldsymbol{a}-\boldsymbol{d}$

The appropriate primary aromatic amine or 1-substituted piperazine (0.01 mole) and $37 \%$ formaldehyde solution $(1.0 \mathrm{~mL})$ were added to a hot solution of 5-(3,4-dimethoxyphenyl)1,3,4-oxadiazole-2 $(3 H)$-thione $3(1.19 \mathrm{~g}, 5.0 \mathrm{mmole})$, in ethanol $(10 \mathrm{~mL})$, and the mixture was stirred at room temperature for $5 \mathrm{~h}$ and allowed to stand overnight. Water $(5 \mathrm{~mL})$ was then added drop-wisely to the reaction mixture with continuous stirring for one hour. The separated precipitate was filtered, washed with water, dried, and crystallized.

3-Anilinomethyl-5-(3,4-dimethoxyphenyl)-1,3,4-oxadiazole-2(3H)-thione 4a. Fine colorless needle crystals. ${ }^{1} \mathrm{H}$ NMR: $\delta 3.84-3.90\left(\mathrm{~m}, 7 \mathrm{H}, \mathrm{OCH}_{3} \& \mathrm{NH}\right), 6.01-6.09\left(\mathrm{~m}, 2 \mathrm{H}, \mathrm{CH}_{2}\right), 6.69(\mathrm{t}$, $1 \mathrm{H}, \mathrm{Ar}-\mathrm{H}, J=7.0 \mathrm{~Hz}), 6.91(\mathrm{~d}, 2 \mathrm{H}, \mathrm{Ar}-\mathrm{H}, J=8.5 \mathrm{~Hz}), 7.08-7.33(\mathrm{~m}, 5 \mathrm{H}, \mathrm{Ar}-\mathrm{H}) .{ }^{13} \mathrm{C}$ NMR: $\delta$ 55.6, $55.8\left(\mathrm{OCH}_{3}\right), 57.9\left(\mathrm{CH}_{2}\right), 108.4,112.1,112.9,114.0,117.9,120.0,129.1,145.3,149.1$, 152.4 (Ar-C), 159.0 (Oxadiazole C5), 175.3 (C=S).

3-[(4-Fluorophenylamino)methyl]-5-(3,4-dimethoxyphenyl)-1,3,4-oxadiazole-2(3H)-thione $\mathbf{4 b}$. Fine colorless needle crystals. ${ }^{1} \mathrm{H}$ NMR: $\delta 3.86-3.90\left(\mathrm{~m}, 7 \mathrm{H}, \mathrm{OCH}_{3} \& \mathrm{NH}\right), 5.49\left(\mathrm{~d}, 2 \mathrm{H}, \mathrm{CH}_{2}\right.$, $J=7.0 \mathrm{~Hz}), 6.89-6.94(\mathrm{~m}, 2 \mathrm{H}, \mathrm{Ar}-\mathrm{H}), 6.99-7.06(\mathrm{~m}, 2 \mathrm{H}, \mathrm{Ar}-\mathrm{H}), 7.21-7.25(\mathrm{~m}, 1 \mathrm{H}, \mathrm{Ar}-\mathrm{H}), 7.32$ $(\mathrm{d}, 1 \mathrm{H}, \mathrm{Ar}-\mathrm{H}, J=1.5 \mathrm{~Hz}), 7.51(\mathrm{~d}, 1 \mathrm{H}, \mathrm{Ar}-\mathrm{H}, J=1.5 \mathrm{~Hz}) .{ }^{13} \mathrm{C}$ NMR: $\delta 55.7,55.8\left(\mathrm{OCH}_{3}\right), 58.3$ $\left(\mathrm{CH}_{2}\right), 108.4,112.1,114.0\left(\mathrm{~d}, J_{\mathrm{C}-\mathrm{F}}=5.0 \mathrm{~Hz}\right), 115.6\left(\mathrm{~d}, J_{\mathrm{C}-\mathrm{F}}=23.0 \mathrm{~Hz}\right), 119.1\left(\mathrm{~d}, J_{\mathrm{C}-\mathrm{F}}=7.0 \mathrm{~Hz}\right)$, 120.1, 141.9, 149.1, 152.4, $155.4\left(\mathrm{~d}, J_{\mathrm{C}-\mathrm{F}}=232.5 \mathrm{~Hz}\right)(\mathrm{Ar}-\mathrm{C}), 159.0$ (Oxadiazole C5), 175.4 $(\mathrm{C}=\mathrm{S})$.

3-[(3-Chlorophenylamino)methyl]-5-(3,4-dimethoxyphenyl)-1,3,4-oxadiazole-2(3H)-thione 4c. Fine colorless needle crystals. ${ }^{1} \mathrm{H}$ NMR: $\delta 3.87-3.88\left(\mathrm{~m}, 7 \mathrm{H}, \mathrm{OCH}_{3} \& \mathrm{NH}\right), 5.50\left(\mathrm{~d}, 2 \mathrm{H}, \mathrm{CH}_{2}\right.$, $J=7.0 \mathrm{~Hz}), 6.73(\mathrm{~d}, 1 \mathrm{H}, \mathrm{Ar}-\mathrm{H}, J=1.5 \mathrm{~Hz}), 6.87(\mathrm{~d}, 1 \mathrm{H}, \mathrm{Ar}-\mathrm{H}, J=1.5 \mathrm{~Hz}), 7.02-7.05(\mathrm{~m}, 1 \mathrm{H}$, Ar-H), 7.17-7.19 (m, 1H, Ar-H), $7.33(\mathrm{~d}, 1 \mathrm{H}, \mathrm{Ar}-\mathrm{H}, J=1.5 \mathrm{~Hz}), 7.51(\mathrm{~d}, 1 \mathrm{H}, \mathrm{Ar}-\mathrm{H}, J=1.5 \mathrm{~Hz})$, 7.55 (t, $1 \mathrm{H}, \mathrm{Ar}-\mathrm{H}, J=7.0 \mathrm{~Hz}) .{ }^{13} \mathrm{C}$ NMR: $\delta 55.6,55.8\left(\mathrm{OCH}_{3}\right), 57.5\left(\mathrm{CH}_{2}\right), 108.4,111.8,112.1$, 112.5, 113.9, 117.5, 120.1, 130.7, 133.8, 147.1, 149.1, 152.4 (Ar-C), 159.1 (Oxadiazole C5), 175.4 $(\mathrm{C}=\mathrm{S})$.

3-[(4-Chlorophenylamino)methyl]-5-(3,4-dimethoxyphenyl)-1,3,4-oxadiazole-2(3H)-thione $4 \mathbf{d}$. Fine colorless needle crystals. ${ }^{1} \mathrm{H}$ NMR: $\delta 3.87-3.88\left(\mathrm{~s}, 7 \mathrm{H}, \mathrm{OCH}_{3} \& \mathrm{NH}\right), 5.49\left(\mathrm{~d}, 2 \mathrm{H}, \mathrm{CH}_{2}\right.$, $J=7.0 \mathrm{~Hz}), 6.93(\mathrm{~d}, 2 \mathrm{H}, \mathrm{Ar}-\mathrm{H}, J=9.0 \mathrm{~Hz}), 7.20-7.22(\mathrm{~m}, 2 \mathrm{H}, \mathrm{Ar}-\mathrm{H}), 7.32(\mathrm{~d}, 1 \mathrm{H}, \mathrm{Ar}-\mathrm{H}$, $J=1.5 \mathrm{~Hz}), 7.42(\mathrm{~d}, 1 \mathrm{H}, \mathrm{Ar}-\mathrm{H}, J=8.0 \mathrm{~Hz}), 7.50(\mathrm{~d}, 1 \mathrm{H}, \mathrm{Ar}-\mathrm{H}, J=1.5,8.0 \mathrm{~Hz}) .{ }^{13} \mathrm{C}$ NMR: $\delta 55.7,55.8\left(\mathrm{OCH}_{3}\right), 57.8\left(\mathrm{CH}_{2}\right), 108.4,112.1,113.9,114.5,120.1,121.5,128.8,144.4,149.1$, 152.4, 159.1 (Oxadiazole C5), $175.4(\mathrm{C}=\mathrm{S})$. 
3-[(2-Nitrophenylamino)methyl]-5-(3,4-dimethoxyphenyl)-1,3,4-oxadiazole-2(3H)-thione 4e. Pale yellow block crystals. ${ }^{1} \mathrm{H}$ NMR: $\delta 3.85-3.89\left(\mathrm{~m}, 7 \mathrm{H}, \mathrm{OCH}_{3} \& \mathrm{NH}\right), 5.77\left(\mathrm{~d}, 2 \mathrm{H}, \mathrm{CH}_{2}\right.$, $J=7.0 \mathrm{~Hz}), 6.89-6.95(\mathrm{~m}, 1 \mathrm{H}, \mathrm{Ar}-\mathrm{H}), 7.16-7.21(\mathrm{~m}, 1 \mathrm{H}, \mathrm{Ar}-\mathrm{H}), 7.44-7.48(\mathrm{~m}, 1 \mathrm{H}, \mathrm{Ar}-\mathrm{H})$, 7.49-7.54 (m, 1H, Ar-H), 7.65-7.70 (m, 1H, Ar-H), 8.16 (d, 1H, Ar-H, J = 1.5 Hz), 8.85-8.91 (m, 1H, Ar-H) ${ }^{13} \mathrm{C}$ NMR: $\delta 55.7,55.8\left(\mathrm{OCH}_{3}\right), 56.6\left(\mathrm{CH}_{2}\right), 108.5,112.1,115.4,117.9,119.8$, 120.0, 125.4, 126.5, 136.6, 142.2, 149.2, 152.5 (Ar-C), 159.3 (Oxadiazole C5), 175.5 (C=S).

3-[(3-Nitrophenylamino)methyl]-5-(3,4-dimethoxyphenyl)-1,3,4-oxadiazole-2(3H)-thione $\mathbf{4 f}$. Pale yellow block crystals. ${ }^{1} \mathrm{H}$ NMR: $\delta 3.86-3.90\left(\mathrm{~m}, 7 \mathrm{H}, \mathrm{OCH}_{3} \& \mathrm{NH}\right), 5.59\left(\mathrm{~d}, 2 \mathrm{H}, \mathrm{CH}_{2}\right.$, $J=7.0 \mathrm{~Hz}), 7.19(\mathrm{~d}, 1 \mathrm{H}, \mathrm{Ar}-\mathrm{H}, J=9.0 \mathrm{~Hz}), 7.32-7.36(\mathrm{~m}, 1 \mathrm{H}, \mathrm{Ar}-\mathrm{H}), 7.45(\mathrm{t}, 1 \mathrm{H}, \mathrm{Ar}-\mathrm{H}$, $J=8.0 \mathrm{~Hz}), 7.51(\mathrm{~d}, 1 \mathrm{H}, \mathrm{Ar}-\mathrm{H}, J=1.5 \mathrm{~Hz}), 7.55(\mathrm{~d}, 1 \mathrm{H}, \mathrm{Ar}-\mathrm{H}, J=1.5 \mathrm{~Hz}), 7.87-7.94(\mathrm{~m}, 2 \mathrm{H}$, Ar-H). ${ }^{13} \mathrm{C}$ NMR: $\delta 55.6,55.8\left(\mathrm{OCH}_{3}\right), 57.4\left(\mathrm{CH}_{2}\right), 106.9,108.4,112.0,112.4,113.9,119.6$, 120.1, 130.2, 146.9, 148.8, 149.1, 152.4 (Ar-C), 159.0 (Oxadiazole C5), 175.5 (C=S).

3-[(4-Nitrophenylamino)methyl]-5-(3,4-dimethoxyphenyl)-1,3,4-oxadiazole-2(3H)-thione $\mathbf{4 g}$. Pale yellow block crystals. ${ }^{1} \mathrm{H}$ NMR: $\delta 3.86-3.90\left(\mathrm{~m}, 7 \mathrm{H}, \mathrm{OCH}_{3} \& \mathrm{NH}\right), 5.61\left(\mathrm{~d}, 2 \mathrm{H}, \mathrm{CH}_{2}\right.$, $J=7.0 \mathrm{~Hz}), 7.07(\mathrm{~d}, 2 \mathrm{H}, \mathrm{Ar}-\mathrm{H}, J=9.0 \mathrm{~Hz}), 7.19(\mathrm{~d}, 1 \mathrm{H}, \mathrm{Ar}-\mathrm{H}, J=8.5 \mathrm{~Hz}), 7.33(\mathrm{~d}, 1 \mathrm{H}, \mathrm{Ar}-\mathrm{H}$, $J=2.0 \mathrm{~Hz}), 7.52(\mathrm{~d}, 1 \mathrm{H}, \mathrm{Ar}-\mathrm{H}, J=2.0 \mathrm{~Hz}), 8.12(\mathrm{~d}, 2 \mathrm{H}, \mathrm{Ar}-\mathrm{H}, J=9.0 \mathrm{~Hz}) .{ }^{13} \mathrm{C}$ NMR: $\delta 55.7$, $55.8\left(\mathrm{OCH}_{3}\right), 56.7\left(\mathrm{CH}_{2}\right), 108.5,112.1,112.5,113.9,120.2,126.0,138.1,149.1,152.1,152.5$ (Ar-C), 159.2 (Oxadiazole C5), 175.5 (C=S).

3-[(2-Trifluoromethylphenylamino)methyl]-5-(3,4-dimethoxyphenyl)-1,3,4-oxadiazole-2(3H)thione $4 \mathrm{~h}$. Colorless prism crystals. ${ }^{1} \mathrm{H}$ NMR: $\delta 3.86-3.90\left(\mathrm{~m}, 7 \mathrm{H}, \mathrm{OCH}_{3} \& \mathrm{NH}\right), 5.61(\mathrm{~d}, 2 \mathrm{H}$, $\left.\mathrm{CH}_{2}, J=6.5 \mathrm{~Hz}\right), 6.89(\mathrm{t}, 2 \mathrm{H}, \mathrm{Ar}-\mathrm{H}, J=8.0 \mathrm{~Hz}), 7.24(\mathrm{~d}, 2 \mathrm{H}, \mathrm{Ar}-\mathrm{H}, J=9.0 \mathrm{~Hz}), 7.31-7.37(\mathrm{~m}$, 2H, Ar-H), 7.50-7.52 (m, 2H, Ar-H). ${ }^{13} \mathrm{C}$ NMR: $\delta 55.7,55.8\left(\mathrm{OCH}_{3}\right), 57.3\left(\mathrm{CH}_{2}\right), 108.5,112.0$, $114.6,115.0,116.7,117.8,119.8,120.1,126.6,133.0,133.7,149.1,152.1\left(\mathrm{Ar}-\mathrm{C} \& \mathrm{CF}_{3}\right), 160.6$ (Oxadiazole C5), $177.2(\mathrm{C}=\mathrm{S})$.

3-[(3-Trifluoromethylphenylamino)methyl]-5-(3,4-dimethoxyphenyl)-1,3,4-oxadiazole-2(3H)thione $4 \mathbf{i}$. White amorphous powder. ${ }^{1} \mathrm{H}$ NMR: $\delta 3.86-3.90\left(\mathrm{~m}, 7 \mathrm{H}, \mathrm{OCH}_{3} \& \mathrm{NH}\right), 5.56(\mathrm{~d}$, $\left.2 \mathrm{H}, \mathrm{CH}_{2}, J=7.5 \mathrm{~Hz}\right), 7.02(\mathrm{~d}, 1 \mathrm{H}, \mathrm{Ar}-\mathrm{H}, J=7.5 \mathrm{~Hz}), 7.17-7.21(\mathrm{~m}, 1 \mathrm{H}, \mathrm{Ar}-\mathrm{H}), 7.31-7.34$ $(\mathrm{m}, 2 \mathrm{H}, \mathrm{Ar}-\mathrm{H}), 7.37-7.42(\mathrm{~m}, 1 \mathrm{H}, \mathrm{Ar}-\mathrm{H}), 7.51(\mathrm{~d}, 1 \mathrm{H}, \mathrm{Ar}-\mathrm{H}, J=1.5 \mathrm{~Hz}), 7.71(\mathrm{t}, 1 \mathrm{H}, \mathrm{Ar}-\mathrm{H}$, $J=7 . \mathrm{Hz}) .{ }^{13} \mathrm{C}$ NMR: $\delta 55.6,55.8\left(\mathrm{OCH}_{3}\right), 57.4\left(\mathrm{CH}_{2}\right), 108.3,109.0,112.0,113.9,115.4,117.0$, 120.1, 123.3, 125.5, 130.1, 146.2, 149.1, 152.4 (Ar-C \& CF 3 ), 159.0 (Oxadiazole C5), 175.5 $(\mathrm{C}=\mathrm{S})$.

3-[(2,4-Difluorophenylamino)methyl]-5-(3,4-dimethoxyphenyl)-1,3,4-oxadiazole-2(3H)-thione 4j. Colorless needle crystals. ${ }^{1} \mathrm{H}$ NMR: $\delta 3.86-3.90\left(\mathrm{~m}, 7 \mathrm{H}, \mathrm{OCH}_{3} \& \mathrm{NH}\right), 5.52(\mathrm{~d}, 2 \mathrm{H}$, $\left.\mathrm{CH}_{2}, J=7.0 \mathrm{~Hz}\right), 6.95-7.04(\mathrm{~m}, 2 \mathrm{H}, \mathrm{Ar}-\mathrm{H}), 7.17-7.21(\mathrm{~m}, 2 \mathrm{H}, \mathrm{Ar}-\mathrm{H}), 7.32(\mathrm{~d}, 1 \mathrm{H}, \mathrm{Ar}-\mathrm{H}$, $J=1.5 \mathrm{~Hz}), 7.49-7.52(\mathrm{~m}, 1 \mathrm{H}, \mathrm{Ar}-\mathrm{H}) .{ }^{13} \mathrm{C}$ NMR: $\delta 55.6,55.8\left(\mathrm{OCH}_{3}\right), 57.9\left(\mathrm{CH}_{2}\right), 104.0(\mathrm{~d}$, $\left.J_{\mathrm{C}-\mathrm{F}}=23.05 \mathrm{~Hz}\right), 108.4,111.0\left(\mathrm{~d}, J_{\mathrm{C}-\mathrm{F}}=19.0 \mathrm{~Hz}\right), 112.0,113.8\left(\mathrm{~d}, J_{\mathrm{C}-\mathrm{F}}=8.5 \mathrm{~Hz}\right), 113.9,120.1$, $\left.130.2\left(\mathrm{~d}, J_{\mathrm{C}-\mathrm{F}}=13.0 \mathrm{~Hz}\right), 149.1,152.4,154.3\left(\mathrm{~d}, J_{\mathrm{C}-\mathrm{F}}=225.5 \mathrm{~Hz}\right), 159.7 \mathrm{~d}, J_{\mathrm{C}-\mathrm{F}}=237.5 \mathrm{~Hz}\right)$ (Ar-C), 159.2 (Oxadiazole C5), $175.4(\mathrm{C}=\mathrm{S})$.

3-[(2,5-Difluorophenylamino)methyl]-5-(3,4-dimethoxyphenyl)-1,3,4-oxadiazole-2(3H)-thione 4k. Colorless needle crystals. ${ }^{1} \mathrm{H}$ NMR: $\delta 3.86-3.90\left(\mathrm{~m}, 7 \mathrm{H}, \mathrm{OCH}_{3} \& \mathrm{NH}\right), 5.51\left(\mathrm{~d}, 2 \mathrm{H}, \mathrm{CH}_{2}\right.$, $J=6.5 \mathrm{~Hz}), 7.02-7.07(\mathrm{~m}, 1 \mathrm{H}, \mathrm{Ar}-\mathrm{H}), 7.12-7.16(\mathrm{~m}, 1 \mathrm{H}, \mathrm{Ar}-\mathrm{H}), 7.18-7.21(\mathrm{~m}, 1 \mathrm{H}, \mathrm{Ar}-\mathrm{H}), 7.33$ $(\mathrm{d}, 1 \mathrm{H}, \mathrm{Ar}-\mathrm{H}, J=1.5 \mathrm{~Hz}), 7.39-7.44(\mathrm{~m}, 1 \mathrm{H}, \mathrm{Ar}-\mathrm{H}), 7.49-7.53(\mathrm{~m}, 1 \mathrm{H}, \mathrm{Ar}-\mathrm{H}) .{ }^{13} \mathrm{C}$ NMR: $\delta$ 55.7, $55.8\left(\mathrm{OCH}_{3}\right), 57.1\left(\mathrm{CH}_{2}\right), 100.5\left(\mathrm{~d}, J_{\mathrm{C}-\mathrm{F}}=27.5 \mathrm{~Hz}\right), 103.2\left(\mathrm{~d}, J_{\mathrm{C}-\mathrm{F}}=7.0 \mathrm{~Hz}\right), 108.4,111.9$, $112.0,114.6,115.6\left(\mathrm{~d}, J_{\mathrm{C}-\mathrm{F}}=11.0 \mathrm{~Hz}\right), 119.8,120.1,149.1,152.1,159.0\left(\mathrm{~d}, J_{\mathrm{C}-\mathrm{F}}=241.0 \mathrm{~Hz}\right)$ (Ar-C), 159.7 (Oxadiazole C5), 175.5 (C=S).

3-[(2,4-Dichlorophenylamino)methyl]-5-(3,4-dimethoxyphenyl)-1,3,4-oxadiazole-2(3H)-thione 41. Fine colorless needle crystals. ${ }^{1} \mathrm{H}$ NMR: $\delta 3.86-3.90\left(\mathrm{~m}, 7 \mathrm{H}, \mathrm{OCH}_{3} \& \mathrm{NH}\right), 5.58(\mathrm{~d}, 2 \mathrm{H}$, $\mathrm{CH} 2, J=7.0 \mathrm{~Hz}), 7.04-7.08(\mathrm{~m}, 1 \mathrm{H}, \mathrm{Ar}-\mathrm{H}), 7.16-7.20(\mathrm{~m}, 1 \mathrm{H}, \mathrm{Ar}-\mathrm{H}), 7.28-7.33(\mathrm{~m}, 2 \mathrm{H}, \mathrm{Ar}-\mathrm{H})$, 7.47-7.52 (m, 2H, Ar-H). ${ }^{13} \mathrm{C}$ NMR: $\delta$ 55.7, $55.8\left(\mathrm{OCH}_{3}\right), 57.4\left(\mathrm{CH}_{2}\right), 108.5,112.0,113.8$, 113.9, 119.1, 120.1, 121.7, 127.9, 128.8, 140.5, 149.1, 152.4 (Ar-C), 159.2 (Oxadiazole C5), 175.4 $(\mathrm{C}=\mathrm{S})$.

5-(3,4-Dimethoxyphenyl)-3-[(4-phenylpiperazin-1-yl)methyl]-1,3,4-oxadiazole-2(3H)-thione 5a. Fine colorless needle crystals. ${ }^{1} \mathrm{H}$ NMR: $\delta$ 2.89-2.99 (m, 4H, Piperazine-H), 3.13-3.23 $\left(\mathrm{m}, 4 \mathrm{H}\right.$, Piperazine-H), $3.89\left(\mathrm{~s}, 6 \mathrm{H}, \mathrm{OCH}_{3}\right), 5.13\left(\mathrm{~s}, 2 \mathrm{H}, \mathrm{CH}_{2}\right), 6.80(\mathrm{t}, 1 \mathrm{H}, \mathrm{Ar}-\mathrm{H}, J=7.0 \mathrm{~Hz})$, 
$6.96(\mathrm{~d}, 2 \mathrm{H}, \mathrm{Ar}-\mathrm{H}, J=8.0 \mathrm{~Hz}), 7.18-7.26(\mathrm{~m}, 3 \mathrm{H}, \mathrm{Ar}-\mathrm{H}), 7.36-7.39(\mathrm{~m}, 1 \mathrm{H}, \mathrm{Ar}-\mathrm{H}), 7.55$ (d, $1 \mathrm{H}, \mathrm{Ar}-\mathrm{H}, J=8.0 \mathrm{~Hz}) .{ }^{13} \mathrm{C}$ NMR: $\delta 48.3,49.6$ (Piperazine-C), 55.7, $55.8\left(\mathrm{OCH}_{3}\right), 69.7\left(\mathrm{CH}_{2}\right)$, 108.5, 112.0, 114.1, 115.7, 119.0, 120.1, 128.9, 149.1, 151.0, 152.3 (Ar-C), 158.7 (Oxadiazole C5), $177.2(\mathrm{C}=\mathrm{S})$.

5-(3,4-Dimethoxyphenyl)-3-\{4-[(4-fluorophenyl)piperazin-1-yl]methyl\}-1,3,4-oxadiazole-2(3H)thione 5b. Colorless needle crystals. ${ }^{1} \mathrm{H}$ NMR: $\delta$ 2.92-2.95 (m, 4H, Piperazine-H), 3.09-3.16 (m, 4H, Piperazine-H), $3.89\left(\mathrm{~s}, 6 \mathrm{H}, \mathrm{OCH}_{3}\right), 5.12\left(\mathrm{~s}, 2 \mathrm{H}, \mathrm{CH}_{2}\right), 6.96-6.98(\mathrm{~m}, 2 \mathrm{H}, \mathrm{Ar}-\mathrm{H})$, 7.05-7.07 (m, 1H, Ar-H), 7.08-7.19 (m, 1H, Ar-H), 7.21 (d, 1H, Ar-H, J = 1.5 Hz), 7.37 (d, 2H, Ar-H, $J=8.0 \mathrm{~Hz}) .{ }^{13} \mathrm{C}$ NMR: $\delta$ 49.1, 49.6 (Piperazine-C), 55.7, $55.8\left(\mathrm{OCH}_{3}\right), 69.7\left(\mathrm{CH}_{2}\right), 108.5$, 112.0, 114.2, $115.3\left(\mathrm{~d}, J_{\mathrm{C}-\mathrm{F}}=21.5 \mathrm{~Hz}\right), 117.4\left(\mathrm{~d}, J_{\mathrm{C}-\mathrm{F}}=7.0 \mathrm{~Hz}\right), 120.1,147.0,149.1,152.3,156.1$ $\left(\mathrm{d}, J_{\mathrm{C}-\mathrm{F}}=236.5 \mathrm{~Hz}\right)($ Ar-C),158.6 (Oxadiazole C5), $177.2(\mathrm{C}=\mathrm{S})$.

5-(3,4-Dimethoxyphenyl)-3-[(4-benzylpiperazin-1-yl)methyl]-1,3,4-oxadiazole-2(3H)-thione 5c. White amorphous powder. ${ }^{1} \mathrm{H}$ NMR: $\delta$ 2.30-2.50 (m, 4H, Piperazine-H), 2.74-2.88 (m, 4H, Piperazine-H), 3.50 (s, 2H, Benzylic $\left.\mathrm{CH}_{2}\right), 3.89\left(\mathrm{~s}, 6 \mathrm{H}, \mathrm{OCH}_{3}\right), 5.05\left(\mathrm{~s}, 2 \mathrm{H}, \mathrm{CH}_{2}\right), 7.19(\mathrm{~d}, 1 \mathrm{H}$, Ar-H, $J=9.0 \mathrm{~Hz}), 7.24-7.41(\mathrm{~m}, 6 \mathrm{H}, \mathrm{Ar}-\mathrm{H}), 7.53(\mathrm{~d}, 1 \mathrm{H}, \mathrm{Ar}-\mathrm{H}, J=8.0 \mathrm{~Hz}) .{ }^{13} \mathrm{C}$ NMR: $\delta 49.5$, 52.3 (Piperazine-C), 55.7, $55.8\left(\mathrm{OCH}_{3}\right), 61.9\left(\right.$ Benzylic $\left.\mathrm{CH}_{2}\right), 69.7\left(\mathrm{CH}_{2}\right), 108.5,112.0,114.3$, 120.0, 127.0, 128.1, 128.8, 137.8, 149.1, 152.2 (Ar-C), 158.6 (Oxadiazole C5), 177.2 (C=S).

5-(3,4-Dimethoxyphenyl)-3-\{4-[(2-trifluorobenzyl)piperazin-1-yl]methyl\}-1,3,4-oxadiazole-2(3H)thione 5d. Colorless needle crystals. ${ }^{1} \mathrm{H}$ NMR: $\delta$ 2.38-2.52 (m, 4H, Piperazine-H), 2.78-2.90 (m, 4H, Piperazine-H), 3.64 (s, 2H, Benzylic $\left.\mathrm{CH}_{2}\right), 3.89\left(\mathrm{~s}, 6 \mathrm{H}, \mathrm{OCH}_{3}\right), 5.06\left(\mathrm{~s}, 2 \mathrm{H}, \mathrm{CH}_{2}\right)$, $7.20(\mathrm{~d}, 1 \mathrm{H}, \mathrm{Ar}-\mathrm{H}, J=8.0 \mathrm{~Hz}), 7.37(\mathrm{~d}, 1 \mathrm{H}, \mathrm{Ar}-\mathrm{H}, J=1.5 \mathrm{~Hz}), 7.47(\mathrm{t}, 1 \mathrm{H}, \mathrm{Ar}-\mathrm{H}, J=7.0 \mathrm{~Hz})$, $7.55(\mathrm{~d}, 1 \mathrm{H}, \mathrm{Ar}-\mathrm{H}, J=8.0 \mathrm{~Hz}), 7.64(\mathrm{t}, 1 \mathrm{H}, \mathrm{Ar}-\mathrm{H}, J=8.0 \mathrm{~Hz}), 7.71(\mathrm{~d}, 1 \mathrm{H}, \mathrm{Ar}-\mathrm{H}, J=8.0 \mathrm{~Hz})$, $7.76(\mathrm{~d}, 1 \mathrm{H}, \mathrm{Ar}-\mathrm{H}, \mathrm{J}=8.0 \mathrm{~Hz}) .{ }^{13} \mathrm{C}$ NMR: $\delta 49.7,52.6$ (Piperazine-C), 55.7, $55.8\left(\mathrm{OCH}_{3}\right), 57.5$ (Benzylic $\left.\mathrm{CH}_{2}\right), 69.8\left(\mathrm{CH}_{2}\right), 108.5,112.0,114.2,118.5,120.1,123.4,125.7,127.3,130.3,132.4$, 137.2, 149.1, 152.3 (Ar-C \& $\left.\mathrm{CF}_{3}\right), 158.6$ (Oxadiazole C5), $177.2(\mathrm{C}=\mathrm{S})$.

\section{Conclusions}

Sixteen 1,3,4-oxadiazole-linked N-Mannich bases namely; 3-arylaminomethyl-5-(3,4dimethoxyphenyl)-1,3,4-oxadiazole-2(3H)-thiones $4 \mathbf{a}-1$ and 3-[(4-substituted piperazin-1yl)-methyl]-5-(3,4-dimethoxyphenyl)-1,3,4-oxadiazole-2(3H)-thiones 5a-d were synthesized and their structures were confirmed by ${ }^{1} \mathrm{H} \mathrm{NMR},{ }^{13} \mathrm{C} N \mathrm{NMR}$ and elemental analysis. The in vitro inhibitory activity of compounds $\mathbf{4 a - 1}$ and $\mathbf{5 a - d}$ was assessed against a panel of standard pathogenic Gram-positive bacteria, Gram-negative bacteria, and the yeastlike pathogenic fungus Candida albicans. Compounds $5 \mathbf{c}$ and $\mathbf{5 d}$ displayed potent broad spectrum antibacterial activities and compounds $\mathbf{4 j}, \mathbf{4 1}, \mathbf{5 a}$, and $\mathbf{5 b}$ showed potent activity against the tested Gram-positive bacteria. The anti-proliferative activity of the compounds was evaluated against prostate cancer (PC3), human colorectal cancer (HCT-116), human hepatocellular carcinoma (HePG-2), human epithelioid carcinoma (HeLa), and human breast cancer (MCF7) cell lines. Compounds $4 \mathbf{1}, \mathbf{5 a}, \mathbf{5 c}$, and $\mathbf{5 d}$ showed potent inhibition of cell proliferation in almost all the tested cancer cell lines. The prepared 1,3,4-oxadiazolelinked N-Mannich bases could be considered good antibacterial and anticancer drug candidates. The biological testing results are considered as preliminary and further investigations, including experimental and theoretical investigations for the exploration of their targets, are required for optimization of their chemotherapeutic activities.

Supplementary Materials: The micro-analytical data $(\mathrm{C}, \mathrm{H}, \mathrm{N}$, and S), the experimental details of the determination of in vitro antimicrobial activity, in vitro anti-proliferative activity, and the NMR spectra can be found online.

Author Contributions: Conceptualization, A.A.E.-E. and A.A.B.M.; methodology, L.H.A.-W., S.S.T., and H.M.H.; validation, H.M.H..; formal analysis, L.H.A.-W., A.A.B.M., and S.S.T.; investigation, L.H.A.-W. and H.M.H.; data curation, H.M.H. and S.S.T.; writing-original draft preparation, A.A.E.E.; writing-review and editing, A.A.E.-E. and A.A.B.M.; supervision, L.H.A.-W., A.A.E.-E.; project administration, A.A.E.-E.; funding acquisition, L.H.A.-W. All authors have read and agreed to the published version of the manuscript. 
Funding: This research was funded by the Deanship of Scientific Research at Princess Nourah bint Abdulrahman University through the Research Groups Program (Grant No. RGP-1442-0010-4).

Conflicts of Interest: The authors declare no conflict of interest.

Sample Availability: Samples of the compounds $\mathbf{4 a - 1}$ and $\mathbf{5 a - d}$ are available from the corresponding author.

\section{References}

1. Verma, G.; Khan, M.F.; Akhtar, W.; Alam, M.M.; Akhter, M.; Shaquiquzzaman, M. A Review Exploring Therapeutic Worth of 1,3,4-Oxadiazole Tailored Compounds. Mini-Rev. Med. Chem. 2019, 19, 477-509. [CrossRef]

2. De Oliveira, C.S.; Lira, B.F.; Barbosa-Filho, J.M.; Lorenzo, J.G.F.; De Athayde-Filho, P.F. Synthetic Approaches and Pharmacological Activity of 1,3,4-Oxadiazoles: A Review of the Literature from 2000-2012. Molecules 2012, 17, 10192-10231. [CrossRef] [PubMed]

3. Majumdar, P.; Pati, A.; Patra, M.; Behera, R.K.; Behera, A.K. Acid Hydrazides, Potent Reagents for Synthesis of Oxygen-, Nitrogen-, and/or Sulfur-Containing Heterocyclic Rings. Chem. Rev. 2014, 114, 2942-2977. [CrossRef] [PubMed]

4. Ogata, M.; Atobe, H.; Kushida, H.; Yamamoto, K. In Vitro Sensitivity of Mycoplasmas Isolated from Various Animals and Sewage to Antibiotics and Nitrofurans. J. Antibiot. 1971, 24, 443-451. [CrossRef] [PubMed]

5. Summa, V.; Petrocchi, A.; Bonelli, F.; Crescenzi, B.; Donghi, M.; Ferrara, M.; Fiore, F.; Gardelli, C.; Paz, O.G.; Hazuda, D.J.; et al. Discovery of Raltegravir, a Potent, Selective Orally Bioavailable HIV-Integrase Inhibitor for the Treatment of HIV-AIDS Infection. J. Med. Chem. 2008, 51, 5843-5855. [CrossRef]

6. Shepard, D.R.; Dreicer, R. Zibotentan for the treatment of castrate-resistant prostate cancer. Expert Opin. Investig. Drugs 2010, 19, 899-908. [CrossRef] [PubMed]

7. McCoull, W.; Addie, M.S.; Birch, A.M.; Birtles, S.; Buckett, L.K.; Butlin, R.J.; Bowker, S.S.; Boyd, S.; Chapman, S.; Davies, R.D.; et al. Identification, optimisation and in vivo evaluation of oxadiazole DGAT-1 inhibitors for the treatment of obesity and diabetes. Bioorganic Med. Chem. Lett. 2012, 22, 3873-3878. [CrossRef]

8. Vardan, S.; Smulyan, H.; Mookherjee, S.; Eich, R. Effects of tiodazosin, a new antihypertensive, hemodynamics and clinical variables. Clin. Pharmacol. Ther. 1983, 34, 290-296. [CrossRef] [PubMed]

9. Schlecker, R.; Thieme, P.C. The synthesis of antihypertensive 3-(1,3,4-oxadiazol-2-yl)phenoxypropanolahines. Tetrahedron 1988, 44, 3289-3294. [CrossRef]

10. Zheng, Z.; Liu, Q.; Kim, W.; Tharmalingam, N.; Fuchs, B.B.; Mylonakis, E. Antimicrobial activity of 1,3,4-oxadiazole derivatives against planktonic cells and biofilm of Staphylococcus aureus. Futur. Med. Chem. 2018, 10, 283-296. [CrossRef] [PubMed]

11. Desai, N.; Bhatt, N.; Somani, H.; Trivedi, A. Synthesis, antimicrobial and cytotoxic activities of some novel thiazole clubbed 1,3,4-oxadiazoles. Eur. J. Med. Chem. 2013, 67, 54-59. [CrossRef]

12. Chen, J.; Luo, Y.; Wei, C.; Wu, S.; Wu, R.; Wang, S.; Hu, D.; Song, B. Novel sulfone derivatives containing a 1,3,4-oxadiazole moiety: Design and synthesis based on the 3D-QSAR model as potential antibacterial agent. Pest Manag. Sci. 2020, 76, 3188-3198. [CrossRef]

13. Xu, H.; Jia, A.; Hou, E.; Liu, Z.; Yang, R.; Yang, R.; Guo, Y. Natural Product-Based Fungicides Discovery: Design, Synthesis and Antifungal Activities of Some Sarisan Analogs Containing 1,3,4-Oxadiazole Moieties. Chem. Biodivers. 2019, 17, e1900570. [CrossRef]

14. Wu, Y.-Y.; Shao, W.-B.; Zhu, J.-J.; Long, Z.-Q.; Liu, L.-W.; Wang, P.-Y.; Li, Z.; Yang, S. Novel 1,3,4-Oxadiazole-2-carbohydrazides as Prospective Agricultural Antifungal Agents Potentially Targeting Succinate Dehydrogenase. J. Agric. Food Chem. 2019, 67, 13892-13903. [CrossRef] [PubMed]

15. Wani, M.Y.; Ahmad, A.; Shiekh, R.A.; Al-Ghamdi, K.J.; Sobral, A.J. Imidazole clubbed 1,3,4-oxadiazole derivatives as potential antifungal agents. Bioorganic Med. Chem. 2015, 23, 4172-4180. [CrossRef]

16. Sonawane, A.D.; Rode, N.D.; Nawale, L.; Joshi, R.R.; Joshi, R.A.; Likhite, A.P.; Sarkar, D. Synthesis and biological evaluation of 1,2,4-triazole-3-thione and 1,3,4-oxadiazole-2-thione as antimycobacterial agents. Chem. Biol. Drug Des. 2017, 90, 200-209. [CrossRef]

17. Desai, N.; Somani, H.; Trivedi, A.; Bhatt, K.; Nawale, L.; Khedkar, V.M.; Jha, P.C.; Sarkar, D. Synthesis, biological evaluation and molecular docking study of some novel indole and pyridine based 1,3,4-oxadiazole derivatives as potential antitubercular agents. Bioorganic Med. Chem. Lett. 2016, 26, 1776-1783. [CrossRef] [PubMed]

18. Benmansour, F.; Eydoux, C.; Querat, G.; de Lamballerie, X.; Canard, B.; Alvarez, K.; Guillemot, J.-C.; Barral, K. Novel 2-phenyl5-[(E)-2-(thiophen-2-yl)ethenyl]-1,3,4-oxadiazole and 3-phenyl-5-[(E)-2-(thiophen-2-yl)ethenyl]-1,2,4-oxadiazole derivatives as dengue virus inhibitors targeting NS5 polymerase. Eur. J. Med. Chem. 2016, 109, 146-156. [CrossRef]

19. Wu, W.; Chen, Q.; Tai, A.; Jiang, G.; Ouyang, G. Synthesis and antiviral activity of 2-substituted methylthio-5-(4-amino-2methylpyrimidin-5-yl)-1,3,4-oxadiazole derivatives. Bioorganic Med. Chem. Lett. 2015, 25, 2243-2246. [CrossRef] [PubMed]

20. Lai, H.; Dou, D.; Aravapalli, S.; Teramoto, T.; Lushington, G.H.; Mwania, T.M.; Alliston, K.R.; Eichhorn, D.M.; Padmanabhan, R.; Groutas, W.C. Design, synthesis and characterization of novel 1,2-benzisothiazol-3(2H)-one and 1,3,4-oxadiazole hybrid derivatives: Potent inhibitors of Dengue and West Nile virus NS2B/NS3 proteases. Bioorganic Med. Chem. 2013, 21, 102-113. [CrossRef] 
21. Benassi, A.; Doria, F.; Pirota, V. Groundbreaking Anticancer Activity of Highly Diversified Oxadiazole Scaffolds. Int. J. Mol. Sci. 2020, 21, 8692. [CrossRef] [PubMed]

22. Glomb, T.; Szymankiewicz, K.; Świątek, P. Anti-Cancer Activity of Derivatives of 1,3,4-Oxadiazole. Molecules $2018,23,3361$. [CrossRef]

23. Akhtar, J.; Siddiqui, A.A.; Khan, A.A.; Ali, Z.; Dewangan, R.P.; Pasha, S.; Yar, M.S. Design, synthesis, docking and QSAR study of substituted benzimidazole linked oxadiazole as cytotoxic agents, EGFR and erbB2 receptor inhibitors. Eur. J. Med. Chem. 2017, 126, 853-869. [CrossRef]

24. Ruel, R.; Thibeault, C.; L’Heureux, A.; Martel, A.; Cai, Z.-W.; Wei, D.; Qian, L.; Barrish, J.C.; Mathur, A.; D’Arienzo, C.; et al. Discovery and preclinical studies of 5-isopropyl-6-(5-methyl-1,3,4-oxadiazol-2-yl)-N-(2-methyl-1H-pyrrolo[2,3-b]pyridin-5yl)pyrrolo[2,1-f][1,2,4]triazin-4-amine (BMS-645737), an in vivo active potent VEGFR-2 inhibitor. Bioorganic Med. Chem. Lett. 2008, 18, 2985-2989. [CrossRef]

25. Altıntop, M.D.; Sever, B.; Çiftçi, G.A.; Turan-Zitouni, G.; Kaplancıklı, Z.A.; Özdemir, A. Design, synthesis, in vitro and in silico evaluation of a new series of oxadiazole-based anticancer agents as potential Akt and FAK inhibitors. Eur. J. Med. Chem. 2018, 155, 905-924. [CrossRef] [PubMed]

26. Sun, J.; Ren, S.-Z.; Lu, X.-Y.; Li, J.-J.; Shen, F.-Q.; Xu, C.; Zhu, H.-L. Discovery of a series of 1,3,4-oxadiazole-2(3 H )-thione derivatives containing piperazine skeleton as potential FAK inhibitors. Bioorganic Med. Chem. 2017, 25, 2593-2600. [CrossRef] [PubMed]

27. Valente, S.; Trisciuoglio, D.; De Luca, T.; Nebbioso, A.; Labella, D.; Lenoci, A.; Bigogno, C.; Dondio, G.; Miceli, M.; Brosch, G.; et al. 1,3,4-Oxadiazole-Containing Histone Deacetylase Inhibitors: Anticancer Activities in Cancer Cells. J. Med. Chem. 2014, 57, 6259-6265. [CrossRef]

28. Pidugu, V.R.; Yarla, N.S.; Bishayee, A.; Kalle, A.M.; Satya, A.K. Novel histone deacetylase 8-selective inhibitor 1,3,4-oxadiazolealanine hybrid induces apoptosis in breast cancer cells. Apoptosis 2017, 22, 1394-1403. [CrossRef] [PubMed]

29. Sun, J.; Li, M.-H.; Qian, S.-S.; Guo, F.-J.; Dang, X.-F.; Wang, X.-M.; Xue, Y.-R.; Zhu, H.-L. Synthesis and antitumor activity of 1,3,4-oxadiazole possessing 1,4-benzodioxan moiety as a novel class of potent methionine aminopeptidase type II inhibitors. Bioorganic Med. Chem. Lett. 2013, 23, 2876-2879. [CrossRef]

30. Mohan, C.D.; Anilkumar, N.C.; Rangappa, S.; Shanmugam, M.K.; Mishra, S.; Chinnathambi, A.; Alharbi, S.A.; Bhattacharjee, A.; Sethi, G.; Kumar, A.P.; et al. Novel 1,3,4-Oxadiazole Induces Anticancer Activity by Targeting NF- $\mathrm{B}$ in Hepatocellular Carcinoma Cells. Front. Oncol. 2018, 8, 42. [CrossRef]

31. Yadav, N.; Kumar, P.; Chhikara, A.; Chopra, M. Development of 1,3,4-oxadiazole thione based novel anticancer agents: Design, synthesis and in-vitro studies. Biomed. Pharmacother. 2017, 95, 721-730. [CrossRef]

32. Ullah, H.; Rahim, F.; Taha, M.; Uddin, I.; Wadood, A.; Shah, S.A.A.; Farooq, R.K.; Nawaz, M.; Wahab, Z.; Khan, K.M. Synthesis, molecular docking study and in vitro thymidine phosphorylase inhibitory potential of oxadiazole derivatives. Bioorganic Chem. 2018, 78, 58-67. [CrossRef]

33. Sun, J.; Zhu, H.; Yang, Z.-M.; Zhu, H.-L. Synthesis, molecular modeling and biological evaluation of 2-aminomethyl-5-(quinolin-2yl)-1,3,4-oxadiazole-2(3H)-thione quinolone derivatives as novel anticancer agent. Eur. J. Med. Chem. 2013, 60, 23-28. [CrossRef] [PubMed]

34. Du, Q.-R.; Li, D.-D.; Pi, Y.-Z.; Li, J.-R.; Sun, J.; Fang, F.; Zhong, W.-Q.; Gong, H.-B.; Zhu, H.-L. Novel 1,3,4-oxadiazole thioether derivatives targeting thymidylate synthase as dual anticancer/antimicrobial agents. Bioorganic Med. Chem. 2013, 21, $2286-2297$. [CrossRef]

35. Haibara, H.; Yamazaki, R.; Nishiyama, Y.; Ono, M.; Kobayashi, T.; Hokkyo-Itagaki, A.; Nishisaka, F.; Nishiyama, H.; Kurita, A.; Matsuzaki, T.; et al. YPC-21661 and YPC-22026, novel small molecules, inhibit ZNF143 activityin vitroandin vivo. Cancer Sci. 2017, 108, 1042-1048. [CrossRef] [PubMed]

36. Abdel-Aziz, M.; Metwally, K.A.; Gamal-Eldeen, A.M.; Aly, O.M. 1,3,4-oxadiazole-2-thione Derivatives; Novel Approach for Anticancer and Tubulin Polymerization Inhibitory Activities. Anti-Cancer Agents Med. Chem. 2015, 16, 269-277. [CrossRef] [PubMed]

37. Ozyazici, T.; Gurdal, E.E.; Orak, D.; Sipahi, H.; Ercetin, T.; Gulcan, H.O.; Koksal, M. Synthesis, anti-inflammatory activity, and molecular docking studies of some novel Mannich bases of the 1,3,4-oxadiazole-2(3 H )-thione scaffold. Arch. der Pharm. 2020, 353, e2000061. [CrossRef] [PubMed]

38. Koksal, M.; Ozkan-Dagliyan, I.; Ozyazici, T.; Kadioglu, B.; Sipahi, H.; Bozkurt, A.; Bilge, S.S. Some Novel Mannich Bases of 5-(3,4-Dichlorophenyl)-1,3,4-oxadiazole-2(3H )-one and Their Anti-Inflammatory Activity. Arch. der Pharm. 2017, $350,1700153$. [CrossRef] [PubMed]

39. Dewangan, D.; Nakhate, K.; Tripathi, D.; Kashyap, P.; Dhongde, H. Synthesis, Characterization and Screening for Analgesic and Anti-inflammatory activities of 2, 5-disubstituted 1,3, 4-oxadiazole derivatives. Anti-Inflammatory Anti-Allergy Agents Med. Chem. 2015, 14, 138-145. [CrossRef]

40. Ma, L.; Xiao, Y.; Li, C.; Xie, Z.-L.; Li, D.-D.; Wang, Y.-T.; Ma, H.-T.; Zhu, H.-L.; Wang, M.-H.; Ye, Y.-H. Synthesis and antioxidant activity of novel Mannich base of 1,3,4-oxadiazole derivatives possessing 1,4-benzodioxan. Bioorganic Med. Chem. 2013, 21, 6763-6770. [CrossRef] 
41. Taha, M.; Ismail, N.H.; Imran, S.; Wadood, A.; Rahim, F.; Saad, S.M.; Khan, K.M.; Nasir, A. Synthesis, molecular docking and $\alpha$-glucosidase inhibition of 5-aryl-2-(6'-nitrobenzofuran-2'-yl)-1,3,4-oxadiazoles. Bioorganic Chem. 2016, 66, 117-123. [CrossRef] [PubMed]

42. Liao, B.-R.; He, H.-B.; Yang, L.-L.; Gao, L.-X.; Chang, L.; Tang, J.; Li, J.-Y.; Li, J.; Yang, F. Synthesis and structure-activity relationship of non-phosphorus-based fructose-1,6-bisphosphatase inhibitors: 2,5-Diphenyl-1,3,4-oxadiazoles. Eur. J. Med. Chem. 2014, 83, 15-25. [CrossRef] [PubMed]

43. Maccioni, E.; Alcaro, S.; Cirilli, R.; Vigo, S.; Cardia, M.C.; Sanna, M.L.; Meleddu, R.; Yanez, M.; Costa, G.; Casu, L.; et al. 3-Acetyl-2,5-diaryl-2,3-dihydro-1,3,4-oxadiazoles: A New Scaffold for the Selective Inhibition of Monoamine Oxidase B. J. Med. Chem. 2011, 54, 6394-6398. [CrossRef]

44. Distinto, S.; Meleddu, R.; Yanez, M.; Cirilli, R.; Bianco, G.; Sanna, M.L.; Arridu, A.; Cossu, P.; Cottiglia, F.; Faggi, C.; et al. Drug design, synthesis, in vitro and in silico evaluation of selective monoaminoxidase B inhibitors based on 3-acetyl-2-dichlorophenyl5-aryl-2,3-dihydro-1,3,4-oxadiazole chemical scaffold. Eur. J. Med. Chem. 2016, 108, 542-552. [CrossRef] [PubMed]

45. Wu, X.; Wang, L.; Hua, Y.; Wang, C.; Batsanov, A.S.; Bryce, M.R. A carbazole-oxadiazole diad molecule for single-emittingcomponent white organic light-emitting devices (WOLEDs). Tetrahedron 2014, 70, 2015-2019. [CrossRef]

46. Kamtekar, K.T.; Monkman, A.P.; Bryce, M.R. Recent Advances in White Organic Light-Emitting Materials and Devices (WOLEDs). Adv. Mater. 2010, 22, 572-582. [CrossRef]

47. Anticancer Research. Anticancer. Res. 2018, 40, 5035-5041. [CrossRef]

48. Karaaslan, C.; Duydu, Y.; Ustundag, A.; Yalcin, C.O.; Kaskatepe, B.; Goker, H. Synthesis \& Anticancer Evaluation of New Substituted 2-(3,4- Dimethoxyphenyl)benzazoles. Med. Chem. 2019, 15, 287-297. [CrossRef]

49. Chitti, S.; Singireddi, S.; Reddy, P.S.K.; Trivedi, P.; Bobde, Y.; Kumar, C.; Rangan, K.; Ghosh, B.; Sekhar, K.V.G.C. Design, synthesis and biological evaluation of 2-(3,4-dimethoxyphenyl)-6 (1,2,3,6-tetrahydropyridin-4-yl)imidazo[1,2-a]pyridine analogues as antiproliferative agents. Bioorganic Med. Chem. Lett. 2019, 29, 2551-2558. [CrossRef] [PubMed]

50. Tan, B.S.; Tiong, K.H.; Muruhadas, A.; Randhawa, N.; Choo, H.L.; Bradshaw, T.D.; Stevens, M.F.; Leong, C.-O. CYP2S1 and CYP2W1 Mediate 2-(3,4-Dimethoxyphenyl)-5-Fluorobenzothiazole (GW-610, NSC 721648) Sensitivity in Breast and Colorectal Cancer Cells. Mol. Cancer Ther. 2011, 10, 1982-1992. [CrossRef] [PubMed]

51. Aly, O.M.; Beshr, E.A.; Maklad, R.M.; Mustafa, M.; Gamal-Eldeen, A. Synthesis, Cytotoxicity, Docking Study, and Tubulin Polymerization Inhibitory Activity of Novel 1-(3,4-Dimethoxyphenyl)-5-(3,4,5-trimethoxyphenyl)-1 H -1,2,4-triazole-3-carboxanilides. Arch. der Pharm. 2014, 347, 658-667. [CrossRef] [PubMed]

52. Ghorab, M.M.; Alsaid, M.S.; Nissan, Y.M.; Ashour, A.E.; Al-Mishari, A.A.; Kumar, A.; Ahmed, S.F. Novel Sulfonamide Derivatives Carrying a Biologically Active 3,4-Dimethoxyphenyl Moiety as VEGFR-2 Inhibitors. Chem. Pharm. Bull. 2016, 64, 1747-1754. [CrossRef]

53. Bansal, S.; Sinha, D.; Singh, M.; Cheng, B.; Tse-Dinh, Y.-C.; Tandon, V. 3,4-Dimethoxyphenyl bis-benzimidazole, a novel DNA topoisomerase inhibitor that preferentially targets Escherichia coli topoisomerase I. J. Antimicrob. Chemother. 2012, 67, $2882-2891$. [CrossRef]

54. Felicetti, T.; Cannalire, R.; Burali, M.S.; Massari, S.; Manfroni, G.; Barreca, M.L.; Tabarrini, O.; Schindler, B.D.; Sabatini, S.; Kaatz, G.W.; et al. Searching for Novel Inhibitors of theS. aureusNorA Efflux Pump: Synthesis and Biological Evaluation of the 3-Phenyl-1,4-benzothiazine Analogues. ChemMedChem 2017, 12, 1293-1302. [CrossRef]

55. Shcherbakov, K.V.; Artemyeva, M.A.; Burgart, Y.V.; Saloutin, V.I.; Volobueva, A.S.; Misiurina, M.A.; Esaulkova, Y.L.; Sinegubova, E.O.; Zarubaev, V.V. 7-Imidazolyl-substituted $4^{\prime}$-methoxy and $3^{\prime}, 4^{\prime}$-dimethoxy-containing polyfluoroflavones as promising antiviral agents. J. Fluor. Chem. 2020, 240, 109657. [CrossRef] [PubMed]

56. El-Emam, A.A.; Al-Deeb, O.A.; Al-Omar, M.; Lehmann, J. Synthesis, antimicrobial, and anti-HIV-1 activity of certain 5-(1adamantyl)-2-substituted thio-1,3,4-oxadiazoles and 5-(1-adamantyl)-3-substituted aminomethyl-1,3,4-oxadiazoline-2-thiones . Bioorganic Med. Chem. 2004, 12, 5107-5113. [CrossRef] [PubMed]

57. Al-Abdullah, E.S.; Sebastian, S.; Al-Wabli, R.I.; El-Emam, A.A.; Panicker, C.; Van Alsenoy, C. Vibrational spectroscopic studies (FT-IR, FT-Raman) and quantum chemical calculations on 5-(Adamantan-1-yl)-3-[(4-fluoroanilino)methyl]-2,3-dihydro-1,3,4oxadiazole-2-thione, a potential chemotherapeutic agent. Spectrochim. Acta Part A. Mol. Biomol. Spectrosc. 2014, 133, 605-618. [CrossRef]

58. Al-Omary, F.A.; Karakaya, M.; Sert, Y.; Haress, N.G.; El-Emam, A.A.; Çırak, Ç. Structural and spectroscopic analysis of 3-[(4phenylpiperazin-1-yl)methyl]-5-(thiophen-2-yl)-2,3-dihydro-1,3,4-oxadiazole-2-thione with experimental (FT-IR, Laser-Raman) techniques and ab initio calculations. J. Mol. Struct. 2014, 1076, 664-672. [CrossRef]

59. Al-Omary, F.A.; Mary, Y.S.; Panicker, C.Y.; El-Emam, A.A.; Al-Swaidan, I.A.; Al-Saadi, A.A.; Van Alsenoy, C. Spectroscopic investigations, NBO, HOMO-LUMO, NLO analysis and molecular docking of 5-(adamantan-1-yl)-3-anilinomethyl-2,3-dihydro1,3,4-oxadiazole-2-thione, a potential bioactive agent. J. Mol. Struct. 2015, 1096, 1-14. [CrossRef]

60. Qadeer, G.; Rama, N.H.; Malik, M.A.; Raftery, J. 3,4-Dimethoxybenzohydrazide. Acta Crystallogr. Sect. E Struct. Rep. Online 2007, 63, 3026. [CrossRef]

61. Abdullah, M.A.; Abuo-Rahma, G.E.-D.A.; Abdelhafez, E.-S.M.; Hassan, H.A.; El-Baky, R.M.A. Design, synthesis, molecular docking, anti- Proteus mirabilis and urease inhibition of new fluoroquinolone carboxylic acid derivatives. Bioorganic Chem. 2017, 70, 1-11. [CrossRef] [PubMed] 
62. Goswami, B.N.; Kataky, J.C.S.; Baruah, J.N.; Nath, S.C. Synthesis of 3,5-disubstituted 1,3,4-oxadiazole-2-thiones as potential fungicidal agents. J. Heterocycl. Chem. 1984, 21, 205-208. [CrossRef]

63. Woods, G.L.; Washington, J.A. Antibacterial susceptibility tests: Dilution and disk diffusion methods. In Manual of Clinical Microbiology; Murray, P.R., Baron, E.J., Pfaller, M.A., Tenover, F.C., Yolken, R.H., Eds.; American Society of Microbiology: Washington, DC, USA, 1995.

64. National Committee for Clinical Laboratory Standards. Approved standard document M-7A.; NCCS: Villanova, PA, USA, 1985.

65. Mosmann, T. Rapid colorimetric assay for cellular growth and survival: Application to proliferation and cytotoxicity assays. J. Immunol. Methods 1983, 65, 55-63. [CrossRef]

66. Berridge, M.; Tan, A. Characterization of the Cellular Reduction of 3-(4,5-dimethylthiazol-2-yl)-2,5-diphenyltetrazolium bromide (MTT): Subcellular Localization, Substrate Dependence, and Involvement of Mitochondrial Electron Transport in MTT Reduction. Arch. Biochem. Biophys. 1993, 303, 474-482. [CrossRef]

67. Tacar, O.; Sriamornsak, P.; Dass, C.R. Doxorubicin: An update on anticancer molecular action, toxicity and novel drug delivery systems. J. Pharm. Pharmacol. 2012, 65, 157-170. [CrossRef] [PubMed] 